\title{
Pin1 facilitates isoproterenol-induced cardiac fibrosis and collagen deposition by promoting oxidative stress and activating the MEK1/2-ERK1/2 signal transduction pathway in rats
}

\author{
XIAN WU, MINGJIANG LI, SU-QIN CHEN, SHA LI and FURONG GUO \\ Department of Cardiology, Hubei Key Laboratory of Cardiology, Cardiovascular Research Institute, \\ Renmin Hospital of Wuhan University, Wuhan, Hubei 430060, P.R. China
}

Received March 16, 2017; Accepted December 15, 2017

DOI: 10.3892/ijmm.2017.3354

\begin{abstract}
Peptidyl-prolyl cis/trans isomerase, NIMAinteracting 1 (Pin1) is a member of a large superfamily of phosphorylation-dependent peptidyl-prolyl cis/trans isomerases, which not only regulates multiple targets at various stages of cellular processes, but is also involved in the pathogenesis of several diseases, including microbial infection, cancer, asthma and Alzheimer's disease. However, the role of Pin1 in cardiac fibrosis remains to be fully elucidated. The present study investigated the potential mechanism of Pin1 in isoprenaline (ISO)-induced myocardial fibrosis in rats. The rats were randomly divided into three groups. Echocardiography was used to evaluate changes in the size, shape and function of the heart, and histological staining was performed to visualize inflammatory cell infiltration and fibrosis. Reverse transcription-quantitative polymerase chain reaction analysis, immunohistochemistry and Picrosirius red staining were used to differentiate collagen subtypes. Additionally, cardiac-specific phosphorylation of mitogen-activated protein kinase kinase 1/2 (MEK1/2) and extracellular-signal regulated protein kinase 1/2 (ERK1/2), and the activities of Pin1 and $\alpha$-smooth muscle actin ( $\alpha$-SMA) and other oxidative stress parameters were estimated in the heart. The administration of ISO resulted in an increase in cardiac parameters and elevated the heart-to-body weight ratio. Histopathological examination of heart tissues revealed interstitial inflammatory cellular infiltrate and disorganized collagen fiber deposition. In addition, lipid peroxidation products and oxidative stress
\end{abstract}

Correspondence to: Professor Mingjiang Li, Department of Cardiology, Hubei Key Laboratory of Cardiology, Cardiovascular Research Institute, Renmin Hospital of Wuhan University, 238 Jiefang Road, Wuhan, Hubei 430060, P.R. China

E-mail: 754851539@qq.com

Key words: peptidyl-prolyl cis/trans isomerase, NIMA-interacting 1, juglone, collagen deposition, cardiac fibrosis, oxidative stress, mitogen-activated protein kinase kinase 1/2-extracellular signal-regulated protein kinase $1 / 2$ signal transduction pathway marker activity in plasma and tissues were significantly increased in the ISO-treated rats. Western blot analysis showed significantly elevated protein levels of phosphorylated Pin1, MEK1/2, ERK1/2 and $\alpha$-SMA in remodeling hearts. Treatment with juglone following intraperitoneal injection of ISO significantly prevented inflammatory cell infiltration, improved cardiac function, and suppressed oxidative stresses and fibrotic alterations. In conclusion, the results of the present study suggested that the activation of Pin1 promoted cardiac extracellular matrix deposition and oxidative stress damage by regulating the phosphorylation of the MEK1/2-ERK1/2 signaling pathway and the expression of $\alpha$-SMA. By contrast, the inhibition of Pin1 alleviated cardiac damage and fibrosis in the experimental models, suggesting that Pin1 contributed to the development of cardiac remodeling in ISO-administered rats, and that the inactivation of Pin1 may be a novel therapeutic candidate for the treatment of cardiovascular disease and heart failure.

\section{Introduction}

Cardiovascular disease and angiocardiopathy have high rates of morbidity and mortality, and have attracted increased attention worldwide due to their associated economic burden, and deterioration in the quality of life of affected patients and their families. Emerging evidence indicates that myocardial fibrosis increases myocardial stiffness, decreases ventricular compliance, and accelerates the occurrence and progression of heart failure in response to external stimulation. During the pathogenesis of ventricular remodeling, nonfunctional fibrous tissue replaces the normal myocardium to preserve myocardial structure and functional integrity (1). However, with the involvement of more intricate neurohumoral factors and pressure or volume overload, the early stages of beneficial adaptation are irreversibly converted into a complete decompensatory period, accompanied by cardiomyocyte necrosis and apoptosis, and the overdeposition of extracellular matrix in the interstitium. Once the above pathological processes are activated, the heart is incapable of returning to its original functional state, even if the initial cause of the injury is abated (2). Although studies have examined the relevant molecular mechanisms underlying myocardial fibrosis, the physiology and pathophysiology 
remain to be fully elucidated. Therefore, investigating the mechanisms underlying the development of cardiac fibrotic conditions is important and beneficial for clinical diagnosis and management.

Substantial experimental and clinical evidence has suggested that multifactorial pathophysiological mechanisms are required for the activation of cardiac fibroblasts and remodeling of the ventricle, including the renin-angiotensin-aldosterone system (RAAS), collagen synthesis and degradation, the inflammatory response and numerous signal transduction pathways, which ultimately results in severe impairments of myocardial structure and function. The sustained activation of cardiac $\beta$-adrenergic receptor ( $\beta$-AR) and the release of catecholamines activate the sympathoadrenal system, which is involved in the development of cardiac remodeling. Additionally, the sustained adrenergic activation of the elevated $\beta$-AR sympathetic nervous system increases cardiac output and impairs systolic function and chamber dilation. Isoproterenol (ISO) is a non-selective $\beta$-AR agonist, which is used widely applied to establish a cardiac remodeling model. Increasing evidence suggests that ISO treatment at high doses supplies short-term increases in myocardial oxidative stress, resulting in the release of proinflammatory cytokines and activation of target protein kinases $(3,4)$. By contrast, studies have shown that various signaling pathways correlate to the pathogenesis of adaptive and maladaptive cardiac remodeling. The mitogen-activated protein kinase (MAPK) signaling pathway is a notable example, which is involved in fibrotic remodeling of the heart, either directly by promoting oxidative stress and mitochondrial dysfunction or indirectly by secreting fibrogenic mediators, which lead to myocardial hypertrophy and fibrosis (5-7). As MAPK family members, activation of the MAPK kinase (MEK)1/2-extracellular signal-regulated kinase (ERK)1/2 signaling pathway involves significant modifications in the emergence and development of the myocardial fibrotic process, by interacting with and phosphorylating protein kinases and transcription factors. It is known that protein phosphorylation is a critical process consisting of complex signaling cascades, which mediate protein structure and folding $(8,9)$ and exert a profound effect on pathophysiological cardiac conditions.

Pin1, as a unique prolyl isomerase, specifically recognizes and transforms phosphorylated serine or threonine immediately to proline (pSer/Thr-Pro). It regulates the catalytic activities of various enzymes by altering their phosphorylation status and subcellular localization, protein interactions and stability (10), and it affects diverse cellular processes at the molecular level (11-13). Previous studies have suggested that Pin1 is associated with the progression of several diseases, including cancer (14), asthma (15), aging (16), neurodegenerative diseases (17) and other autoimmune diseases (18). Emerging evidence has shown that Pin1 may be one of the most unique and specific factors correlating to the pathogenesis of various heart diseases. A study by Toko et al (19) demonstrated that Pin1-knockout mice (Pin1-KO) showed alleviated pathological hypertrophy, reserved cardiac function and prolonged survival rates in an experimental group, in which mice underwent abdominal aortic constriction, compared with the sham. Toko et al (19) also demonstrated that Pin1 was mainly involved in cardiac hypertrophy and injury caused by pressure overload, and that the inhibition of Pin1 provided beneficial effects by mediating myocardial regeneration and antagonizing cellular senescence (20). However, the specific mechanisms by which Pin1 mediates myocardial fibrosis following exposure to stress stimuli, including ISO, remains to be fully elucidated. The aim of the present study was to examine whether Pin1 facilitated ISO-induced structural remodeling by accelerating oxidative stress and collagen deposition through the MEK1/2-ERK1/2 signaling pathway in rats.

\section{Materials and methods}

Chemicals and reagents. Juglone was purchased from Macklin Biochemical. (Shanghai, China); ISO, and malondialdehyde (MDA; A3920) and superoxide dismutase (SOD; S9693) kits were purchased from Nanjing Jiancheng Bioengineering Institute (Nanjing, China). The antibodies used were as follows: Phosphorylated (p)ERK (4370), pMEK (9154; Cell Signaling Technology, Inc., Danvers, MA, Germany), $\alpha$-smooth muscle actin ( $\alpha$-SMA; TDY210; TDY Biotech Co.,Beijing,China), glyceraldehyde 3-phosphate dehydrogenase (GAPDH) (ab37168; Abcam, Cambridge, MA, USA) and Pin1 (SAB4504052; Sigma-Aldrich, Merck Millipore), goat anti-rabbit immunoglobulin G-horseradish-peroxidase (IgG-HRP) and goat anti-mouse IgG-HRP (074-1506, 074-1806) were from Kirkegaard and Perry Laboratories, Inc., (Gaithersburg, MD, USA), collagen I and III (ab34710, ab6310, respectively) were from Abcam. Goat anti-rabbit fluorescein isothiocyanate (FITC; AS-1110), goat anti-mouse FITC (AS-1112), goat anti-rabbit CY3 (AS-1109), goat anti-mouse CY3 (AS-1111) and donkey anti-mouse CY3 (AS-1113) were from Aspen Biological (Wuhan, China). 5-Hydroxy-1,4-naphthoquinone (H47002) was from Sigma-Aldrich (Merck Millipore).

Animals. Male Sprague-Dawley rats of 7-8 weeks age, weighing 180-200 g, were purchased from the Center for Disease Control and Prevention of Hubei Province (Hubei, China). The rats were maintained at $22-25^{\circ} \mathrm{C}$ under a $12-\mathrm{h}$ light/dark cycle with a relative humidity of $40-70 \%$. The animals had free access to standard laboratory chow and drinking water. All experiments were approved by the Animal Care and Use Committee of Renmin Hospital of Wuhan University (Wuhan, China) and conformed to the Guidelines for the Care and Use of Laboratory Animals prepared by the National Academy of Sciences and published by the National Institutes of Health (21).

Experimental design. A total of 36 rats were divided randomly into three groups (12 in each group): In the control group (vehicle control), the rats did not receive ISO or juglone; in the ISO group, the rats received an intraperitoneal injection of ISO $(5 \mathrm{mg} / \mathrm{kg})$ without juglone every day for 3 weeks; in the ISO+juglone group, rats received intraperitoneal injection of ISO and juglone at 5 and $3 \mathrm{mg} / \mathrm{kg}$, respectively. The ISO and juglone were dissolved in saline. The rats in the control group were handled similarly, but with an intraperitoneal injection of the same volume of saline. At the end of the experiment, the rats were sacrificed, and blood samples were collected and centrifuged $\left(4,390 \mathrm{x} \mathrm{g}, 15 \mathrm{~min}\right.$ at $\left.37^{\circ} \mathrm{C}\right)$ to separate the serum, which was stored at $-20^{\circ} \mathrm{C}$ for subsequent biochemical assays. 
The hearts were dissected and weighed to calculate the heart weight/body weight ( $\mathrm{HW} / \mathrm{BW}$; mg/g), and the left ventricle of the heart tissues was snap-frozen in liquid nitrogen and stored at $-80^{\circ} \mathrm{C}$ until biochemical analysis or fixed in paraformaldehyde for histological analysis.

Echocardiography. Transthoracic echocardiography measurements (vivid4; GE Healthcare Life Sciences, Chalfont, UK) were performed under anesthesia with intraperitoneal administration of chloral hydrate $(30 \mathrm{mg} / \mathrm{kg})$. At the level of the papillary muscles, using M-mode echocardiography, morphometric and functional parameters, including left ventricular wall thickness (LVPW), left ventricular septum thickness (IVS), left ventricular ejection fraction (EF\%) and left ventricular fractional shortening (FS\%), were obtained in the short-axis view. All echocardiographic parameters were determined based on the average of three consecutive cardiac cycles.

Histopathological analysis. The heart tissues were washed with saline solution (10092-18; Jinuo Co, Hangzhou, China), fixed in 4\% paraformaldehyde (As1018; Aspen Biological; $\geq 24 \mathrm{~h}$ at $37^{\circ} \mathrm{C}$ ), and then processed using analytical grade ethanol and xylene. The paraffinized sections (4-5 $\mu \mathrm{m}$ thick) were stained with $\mathrm{H} \& \mathrm{E}$ (hematoxylin, 3-8 min at $37^{\circ} \mathrm{C}$ and eosin, $1-3 \mathrm{~min}$ at $37^{\circ} \mathrm{C}$ ) to visualize inflammatory cell infiltration, cardiomyocyte necrosis and apoptosis, and fibrosis in myocardial tissue. As an efficacious histochemical stain for collagen detection in tissue sections, Picrosirius red (PSR; AS1067; Aspen Biological was used to differentiate the collagen types. In addition, using Masson's trichrome staining $\left(1-3 \mathrm{~min}\right.$ at $\left.37^{\circ} \mathrm{C}\right)$, the collagen volume fraction $(\mathrm{CVF})$, and the interstitial collagen volume fraction were examined to quantify the degree of fibrosis. Cardiomyocytes were stained red and fibrous tissues were stained in blue by Masson's Trichrome staining. The sections were then examined by light microscopy and images were captured at $\times 40$ and $x 200$ magnification. The percentages of fibrosis in the heart were analyzed using Image-Pro Plus software (version 6.0; Media Cybernetics, Inc., Rockville, MD, USA) and the captured images and their average values were assessed.

Immunofluorescence assay. The experimental steps were as follows: Sections of myocardial tissue were fixed with $4 \%$ paraformaldehyde $(4 \mu \mathrm{m})$ for $\geq 24 \mathrm{~h}$ at $37^{\circ} \mathrm{C}$, followed by washing with phosphate-buffered saline (PBS) for $30 \mathrm{~min}$, and then blocked for $1 \mathrm{~h}$ with $\mathrm{PBS}\left(37^{\circ} \mathrm{C}\right)$. Following incubation with the primary antibody (anti-Pin 1 antibody, 1:25) overnight at $4^{\circ} \mathrm{C}$, the cardiac tissues were incubated with secondary antibody (goat anti-mouse CY3, 1:50) for $1 \mathrm{~h}$ at room temperature. The nuclei were visualized with DAPI $\left(10 \mathrm{~min}, 37^{\circ} \mathrm{C}\right.$; blue $)$. Images were captured by fluorescence microscopy (550-750 nm; IX51; Olympus Corp., Tokyo, Japan) using a 200X objective.

To further evaluate the collagen types, the paraffinized sections were stained using standard immunofluorescence staining techniques. First, the sections were prepared, dried, dewaxed, hydrated and repaired. Second, the sections were washed in PBS, sealed with $10 \%$ sheep serum (Gibco; Thermo Fisher Scientific, Inc., Waltham, MA, USA) for $1 \mathrm{~h}$ at $37^{\circ} \mathrm{C}$ and incubated with primary antibodies (collagen I
1:150; collagen III 1:200) at $4^{\circ} \mathrm{C}$ overnight. The sections were washed again with PBS and incubated with the corresponding secondary antibodies (goat anti-rabbit CY3 1:50; goat anti-mouse FITC, 1:50) for $1 \mathrm{~h}$ at $37^{\circ} \mathrm{C}$. The cells were immunostained with antibodies against collagen type I (red) and collagen type III (green), and the nuclei were labeled with DAPI (10 $\min , 37^{\circ} \mathrm{C}$; blue).

Fluorogenic probe dihydroethidium (DHE) assay and biochemical estimations. Considering the important function of oxidative stress in ventricular remodeling, the present study examined the contribution of Pin1 to ISO-mediated oxidative stress using a DHE assay and biochemical analyses. The fluorogenic probe solution was diluted 1:10,000 according to the manufacturer's protocols and protected from the light. The heart tissues were isolated, washed in PBS and frozen at $-80^{\circ} \mathrm{C}$. Following sectioning with a Microtome-Cryostat (CM1900; Leica Microsystems, Inc., Buffalo Grove, IL, USA), the frozen heart sections were incubated with DHE solution for $30 \mathrm{~min}$ at room temperature to allow incorporation of the DHE into the membrane. Oxidative stress was indicated by red staining under a fluorescence microscope (550-750 nm; IX51; Olympus Corp.). A total of 10 fluorescent images per section of DHE staining were captured using a confocal microscope (magnification, x40 and x200). Additionally, the activity of SOD and levels of MDA were quantified in the serum using detection kits according to the manufacturer's protocols.

Reverse transcription-quantitative polymerase chain reaction $(R T-q P C R)$ analysis. Total RNA was extracted from left ventricle tissues with TRIzol reagent (15596-026; Invitrogen; Thermo Fisher Scientific, Inc.) and reverse-transcribed to cDNA according to the manufacturer's protocols. The synthesis of the first cDNA was carried out in two stages: The first reaction solution (5 g DNA eraser buffer $2.0 \mu \mathrm{l}$, gDNA eraser $1.0 \mu \mathrm{l}$, RNA $1.0 \mu \mathrm{g}$, RNase Free $\mathrm{dH}_{2} \mathrm{O}$ up to $10.0 \mu \mathrm{l}$ ) was prepared on ice to remove genome DNA; Primescript RT enzyme mix I $(1.0 \mu \mathrm{l})$, RT primer mix $(1.0 \mu \mathrm{l}), 5^{*}$ Primescript buffer $2(4.0 \mu \mathrm{l})$ and RNase Free $\mathrm{dH}_{2} \mathrm{O}(4.0 \mu \mathrm{l})$ was then added to the first reaction solution for reverse transcription PCR. RT-qPCR analysis of the mRNA levels of Pin1, collagen type I and III was performed using the Toyobo First Strand cDNA synthesis kit (ReverTra Ace- $\alpha$-; FSK-100; Toyobo Co., Osaka, Japan). The qPCR pre-denaturation was performed at $95^{\circ} \mathrm{C}$ for $1 \mathrm{~min}$; followed by 40 cycles at $95^{\circ} \mathrm{C}$ for $15 \mathrm{sec}, 58^{\circ} \mathrm{C}$ for $20 \mathrm{sec}$ and $72^{\circ} \mathrm{C}$ for $45 \mathrm{sec}$. The melting curve was performed at a temperature ranging between 60 and $95^{\circ} \mathrm{C}\left(1^{\circ} \mathrm{C}\right.$ every $20 \mathrm{sec})$ using the SYBR ${ }^{\circledR}$ Premix Ex Taq ${ }^{\mathrm{TM}}$ kit $(\mathrm{RR} 420 \mathrm{~A}$; Takara Bio, Inc., Otsu, Japan) on a StepOne ${ }^{\text {TM }}$ Real-Time PCR apparatus (Thermo Fisher Scientific, Inc.). Each experiment was performed in triplicate. The following gene primers (Invitrogen; Thermo Fisher Scientific, Inc.) were used: Pin1, forward, 5'-CTGGTGAAGCACACCAATCT-3' and reverse, 5'-GATGCCTGAATCCGTGAACAC-3'; collagen I, forward, 5'-CCGTGACCTCAAGATGTGCC-3' and reverse, 5'-GAA CCTTCGCTTCCACTCG-3'; collagen III, forward 5'-TGT CCACAGCCTTCTACACCT-3' and reverse, 5'-TAGCCACCC ATTCCTCCG-3'; GAPDH, forward, 5'-CGCTAACATCAA ATGGGGTG-3' and reverse, 5'-TTGCTGACAATCTTGAGG GAG-3'. GAPDH was used as the invariant control (22). 
Western blot analysis. Western blot analysis was performed to assess the protein expression levels of Pin 1, $\alpha$-SMA, pMEK1/2 and pERK1/2 in the myocardium in the different experimental groups. Left ventricle myocardial tissue was homogenized in an appropriate volume of ice-cold radioimmunoprecipiation lysis buffer (AS1004; Aspen Biological). Subsequently, the supernatant was collected following centrifugation for $3,600 \times \mathrm{g}$ for $5 \mathrm{~min}$ at $4^{\circ} \mathrm{C}$. The protein concentrations of the samples were determined using the Bicinchoninic Acid protein assay kit (AS1086; Aspen Biological). Equal quantities of samples $(40 \mu \mathrm{g})$ were loaded onto gels for sodium dodecyl sulfate-polyacrylamide gel electrophoresis (SDS-PAGE) (10\% for GAPDH, $\alpha$-SMA, pMEK1/2 and pERK1/2; $12 \%$ for Pin 1 ) and blotted onto polyvinylidene fluoride membranes, which were blocked with $5 \%$ skim milk for $1 \mathrm{~h}$ at $37^{\circ} \mathrm{C}$ to reduce any nonspecific binding. The membranes were then incubated with various primary antibodies against $\alpha$-SMA $(1: 5,000)$, Pin1 $(1: 1,000)$, pMEK1/2 $(1: 1,500)$ and pERK1/2 $(1: 1,500)$ overnight at $4^{\circ} \mathrm{C}$. Following washing for three times with Tris-buffered saline containing $0.1 \%$ Tween-20, the membranes were treated with either HRP-conjugated goat anti-rabbit or goat anti-mouse secondary antibodies at 1:10,000 dilutions for $1 \mathrm{~h}$ at room temperature to detect immunoreactive bands. The optical density was determined with AlphaEaseFC software v4.00 (Alpha Innotech, San Leandro, CA, USA) and an enhanced chemiluminescence kit (AS1059; Aspen Biological) was used to analyze the chemiluminescence of the blots. GAPDH was used as the loading control.

Statistical analysis. All experiments were repeated at least three times with consistent results and SPSS 17.0 (SPSS, Inc., Chicago, IL, USA) was used for statistical analyses. The data are presented as the mean \pm standard deviation. One-way analysis of variance was used to compare multiple groups, followed by Tukey's post hoc analysis. Independent sample t-tests were used to compare two groups as appropriate. $\mathrm{P}<0.05$ was considered to indicate a statistically significant difference.

\section{Results}

Pinl aggravates histopathological changes in cardiac fibrosis induced by ISO in vivo. In the present study, ISO was selected to establish cardiac remodeling. ISO is a non-selective $\beta$-AR agonist used to pharmacologically activate the actions of cardiac myocytes and for treatment of chronic heart failure, and is widely used to establish cardiac remodeling models by altering signal transduction pathways and activating $\beta$-AR. The subcutaneous administration of ISO, which mimics $\beta$-AR activity, can produce myocardial necrosis (23). Continuous sympathetic activation in the myocardium promotes the increase of left ventricular mass and cardiac noradrenaline. Catecholamines activate $\beta$-AR through the classic Smads signaling pathway and promote the transfer of nuclear factor- $\kappa \mathrm{B}$ to the cells, which accelerates the progression of myocardial fibrosis. Increasing evidences suggests that ISO treatment at high doses increases myocardial oxidative stress and pro-inflammatory cytokine synthesis, and stimulates MAPKs (3). Cardiac fibrosis induced by ISO is a reliable, consistent and well-characterized prototype, correlated with arrhythmias, myocyte loss and fibrosis with advancement to heart failure. Therefore, it is possible to use this method to establish a model of fibrotic remodeling in rats.

To investigate the variations in histopathological and morphologic alterations in the experiment intervention groups, $\mathrm{H} \& \mathrm{E}$ and Masson's trichrome staining were applied to evaluate cellular inflammatory infiltration, cardiomyocyte necrosis, and apoptosis and interstitial fibrosis in the different groups. As shown in Fig. 1A, substantial inflammatory cellular infiltrate in the interstitium and disorganized cardiac muscle fibers were observed in the ISO group, compared with the control group. However, these effects were reduced in the group treated with juglone following ISO. In addition, compared with the control group, the myocardial tissue was arranged irregularly and was congested with large quantities and disordered collagen fibers in the ISO group (Fig. 1B). A marked decrease in fibrotic connective tissue was also noted in the ISO+juglone group, which was consistent with the previous H\&E staining results. To accurately differentiate the differences in the degree of fibrosis in the myocardium in response to ISO, the percentages of fibrotic tissue was determined in the captured images using Image-Pro plus software (version 6.0). The CVF and interstitial collagen volume fraction were significantly increased in the ISO group $(\mathrm{P}<0.01)$. However, juglone significantly reduced the CVF $(\mathrm{P}<0.01)$ and interstitial collagen volume fraction $(\mathrm{P}<0.05)$ following ISO injection in rats (Fig. 1C).

Pinl accelerates cardiac dysfunction caused by ISO in vivo. To probe the underlying association between Pin1 and cardiac function, echocardiography was used to evaluate changes in the size, shape and function of the heart. Compared with the control group, the group of rats treated with ISO suffered marked injury, as indicated by the sharp decrease in LVPW, IVS, EF\%, FS\% and the ratio of $\mathrm{HW} / \mathrm{BW}$ (Fig. 2A and B). The macroscopic image shown in Fig. 2A approximately reflects the significant dilation of the left ventricle in the ISO group. Compared with the control group, the ratio of $\mathrm{HW} / \mathrm{BW}$ was lower in the ISO-treated rats $(\mathrm{P}<0.01)$. No statistically significant differences were found in $\mathrm{HW} / \mathrm{BW}$ between the wild-type (WT) controls and rats that treated with juglone following ISO injection, with the exception of a marginal decrease. According to the statistics presented in Fig. 2B, the $\mathrm{EF} \%$ in the control group was maintained at $84.97 \pm 0.47 \%$, but decreased to $64.32 \pm 1.54 \%$ in the ISO rats and increased to almost $75.54 \pm 0.99 \%$ in the ISO+juglone group $(\mathrm{P}<0.05)$; IVS was significantly lower in the ISO group $(2.7 \pm 0.2 \mathrm{~mm})$, compared with the other experimental groups. By contrast, FS\% decreased from $48.45 \pm 0.49 \%$ in the control group to $\sim 31.03 \pm 1.08 \%$ in the ISO group $(\mathrm{P}<0.01)$, and then increased again to $\sim 38.21 \pm 0.66 \%$ in the ISO+juglone group. Similarly, the overall tendency observed for LVPW mimicked the fractional shortening in the bar graph (Fig. 2B). Based on the aforementioned results, it was concluded that the subcutaneous injection of ISO in rats successfully induced myocardial injury and that juglone improved the impaired left ventricular structure induced by ISO in vivo.

Effect of Pinl on the deposition and mRNA expression of collagen I and III in ISO-induced rats. Fibrogenesis is characterized by the excessive accumulation and deposition of extracellular matrix (ECM) proteins. The quality and 

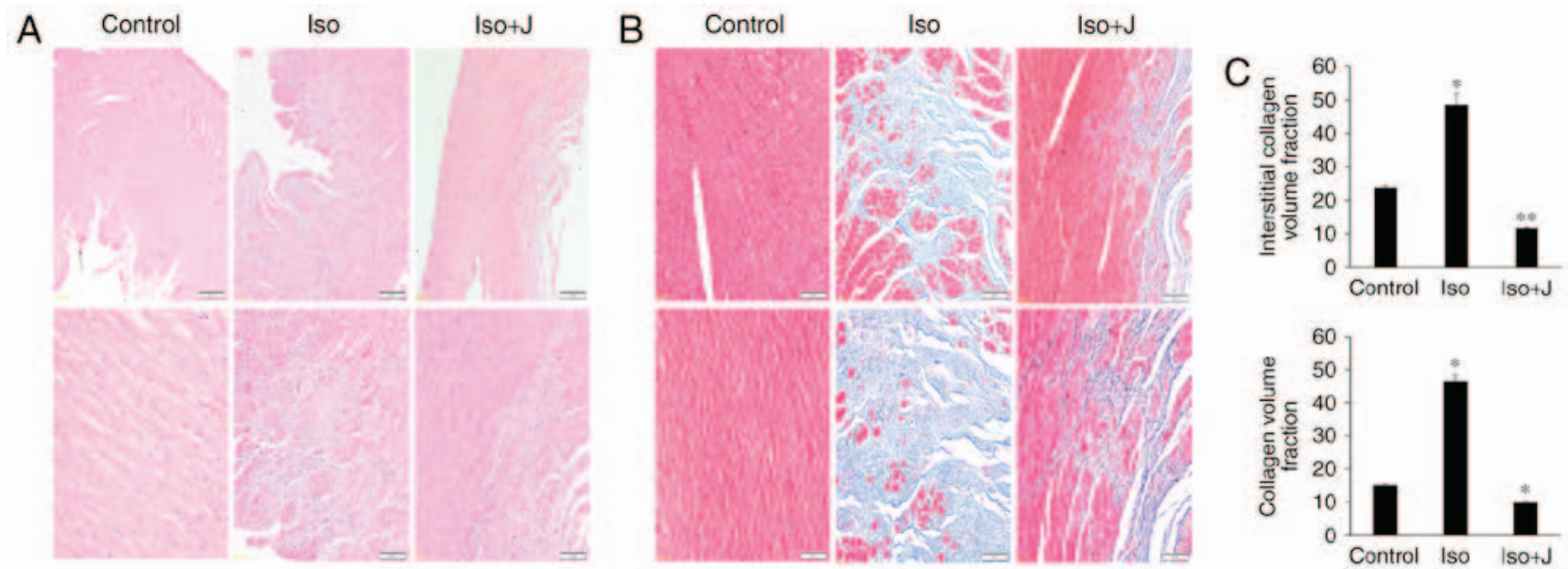

Figure 1. H\&E and Masson's trichrome staining of myocardial tissues. (A) Histological analysis and pathological changes in cardiac tissue. Representative images of H\&E-stained fields (magnification, $x 40$, top panels and x200, bottom panels) are shown for the left ventricles of rats in the control group and rats treated with ISO+juglone or ISO alone. (B) Representative Masson's trichrome staining in the different groups. (C) Quantitative analysis of the myocardial interstitial collagen volume fraction ( $\left(\mathrm{P}<0.01\right.$ and ${ }^{* *} \mathrm{P}<0.05$ vs. control) and collagen volume fraction $(" \mathrm{P}<0.0$ vs. control) in the left ventricles of the experimental and control groups. Control, normal rat. ISO, rat intraperitoneally injected with ISO $(5 \mathrm{mg} / \mathrm{kg})$. ISO+J, ISO rat intraperitoneally injected with juglone $(3 \mathrm{mg} / \mathrm{kg})$. Data is presented as the mean \pm standard error of the mean from at least three independent experiments. H\&E, hematoxylin and eosin; ISO, isoprenaline; J, juglone.

A

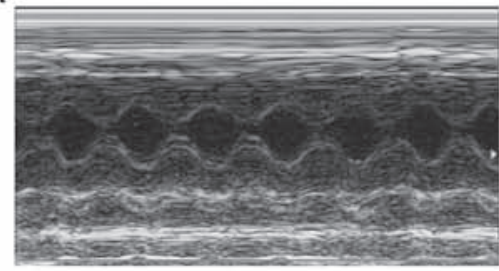

Iso

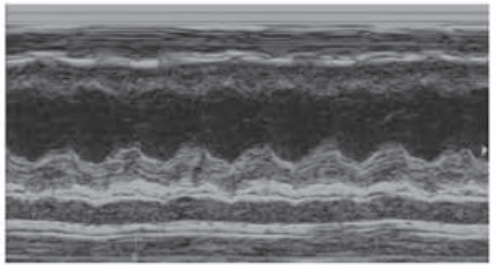

Iso+J

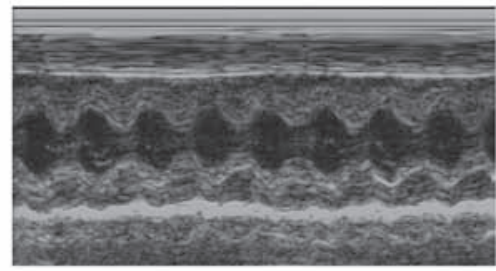

B
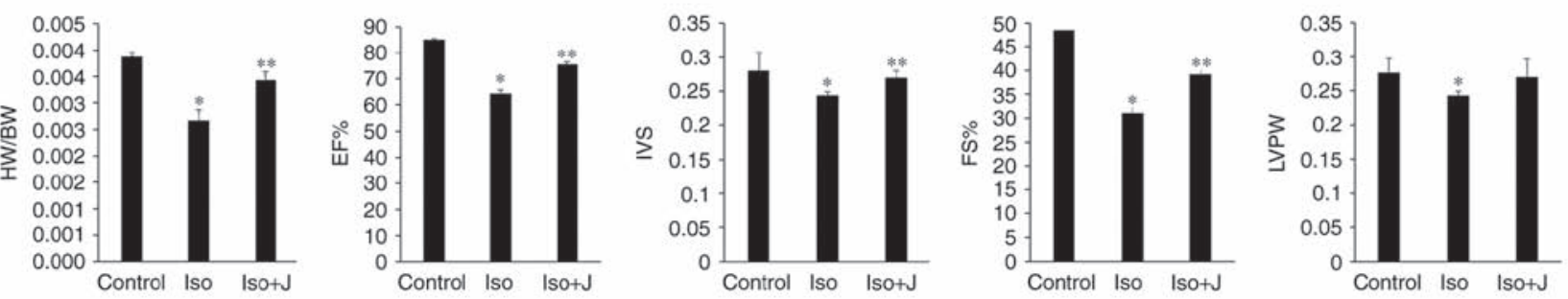

Figure 2. Assessment of cardiac structure and function. (A) Representative M-mode echocardiograms in the control, ISO and ISO+J groups. (B) Bar charts from the left show the HW/BW ratio, EF\%, IVS, FS\%, and LVPW. " $\mathrm{P}<0.01$ ISO group vs. control group; ${ }^{* * *} \mathrm{P}<0.05$ ISO group vs. ISO+J group for EF\%, IVS and FS\%; ${ }^{P}<0.05$ ISO group vs. control group for LVPW. Control, normal rat. ISO, rat intraperitoneally injected with ISO (5 mg/kg); ISO+J, ISO rat intraperitoneally injected with juglone $(3 \mathrm{mg} / \mathrm{kg}$ ). Data are presented as the mean \pm standard error of the mean from at least three different independent experiments. ISO, isoprenaline; J, juglone; EF\%, left ventricular ejection fraction; IVS, interventricular septal thickness; FS\%, fractional shortening; LVPW, left ventricular posterior wall thickness.

organization of collagen fibers significantly affects the pathogenesis of cardiac fibrosis and the tensile strength of interstitial tissue. ECM proteins, including collagen I and collagen III, which are the most abundant collagen types in cardiac tissue and predominantly contribute to interstitial fibrosis, respond to various pathological stimuli. Therefore, PSR staining, immunofluorescence assays and RT-qPCR techniques are used to detect the deposition and mRNA transcription of collagen I and III, reflecting the association between Pin1 and alterations in collagen organization. As shown in Fig. 3A, regular and thin collagen fibers distributed in the myocardium of normal rats were stained red in the PSR-stained sections. Compared with the vehicle group, the ISO-treated rat tissues showed excessive interstitial and perivascular collagen deposition, and fragmented and disordered myocardial muscle bundles (Fig. 3A), whereas juglone treatment significantly attenuated the deposition of collagen $(\mathrm{P}<0.05)$. Additionally, an immunofluorescence technique devised to further differentiate collagen types among the different groups (Fig. 3B) showed significantly higher expression levels of collagen I than III in the rats treated with ISO+juglone, compared with those in rats treated with ISO only.

In the present study, the percentage of the collagen fibers in the ISO group increased, compared with that in the control group $(\mathrm{P}<0.01)$. By contrast, the percentage of fibrous tissue, including collagen I and III, and total collagen in the ISO+juglone group was significantly lower, compared with that in the ISO group $(\mathrm{P}<0.01)$. In addition, the varying 

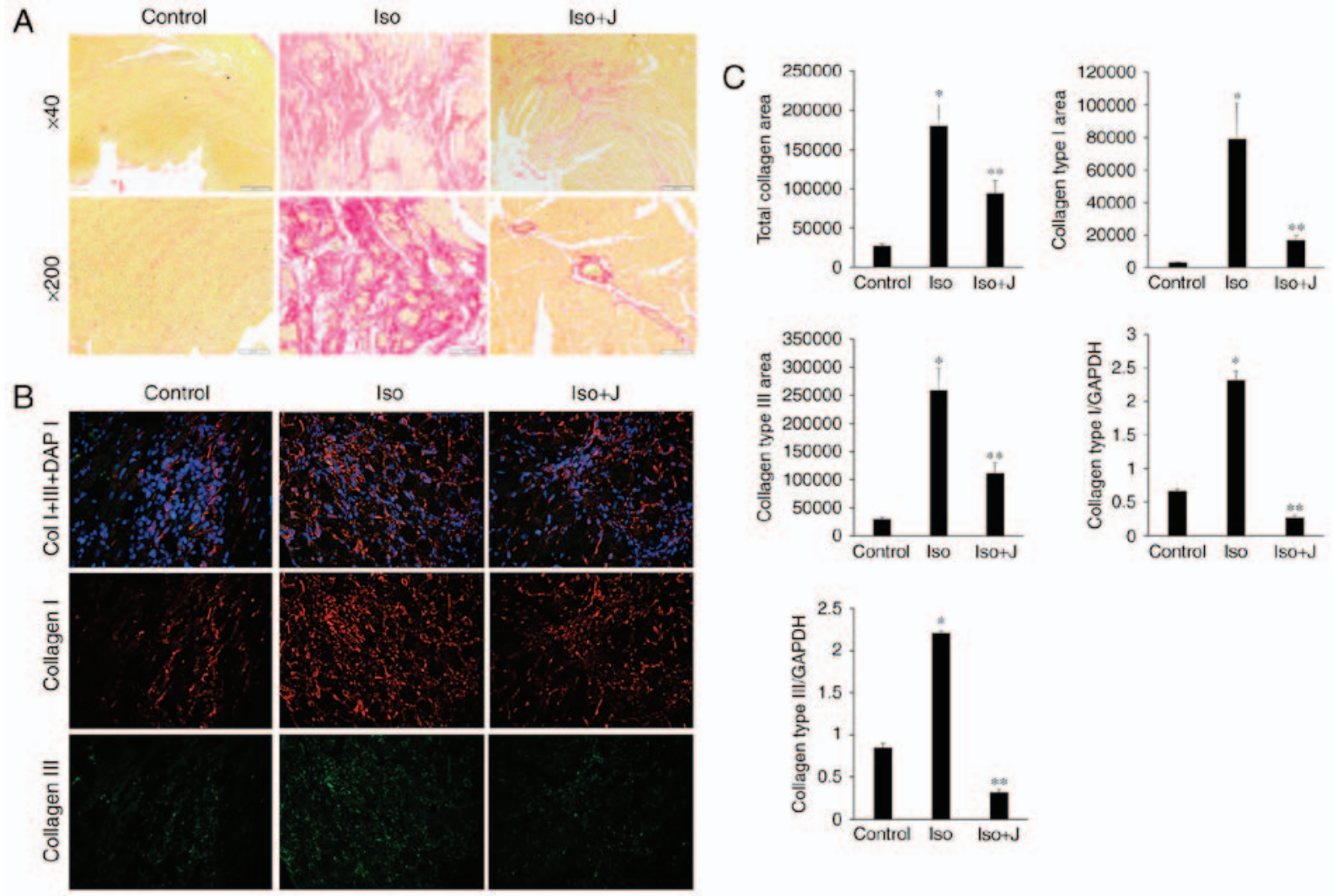

Figure 3. Qualitative and quantitative analysis of collagen accumulation. (A) Picrosirius red staining of histological sections of the left ventricle to assess collagen deposition in different groups (magnification, $x 40$, top panels and $\times 200$, bottom panels). (B) Immunofluorescent staining of collagen I, collagen III and total collagen proteins. Collagen I is stained red, collagen III is stained green, nuclei are stained blue with 4,6-diamidino-2-phenylindole (magnification, x200). (C) Bar graphs shows the results of the quantitative analysis of the expression of collagen I, collagen III, total collagen proteins, collagen deposition, and mRNA levels of collagen type I and collagen type III, which were measured using an image analysis system. ${ }^{*} \mathrm{P}<0.01$ ISO group vs. control group; ${ }^{* *} \mathrm{P}<0.01$ ISO group vs. ISO+J group control, normal rat. ISO, rat intraperitoneally injected with ISO $(5 \mathrm{mg} / \mathrm{kg})$. ISO+J: ISO rat intraperitoneally injected with juglone $(3 \mathrm{mg} / \mathrm{kg}$ ). Data are presented as the mean \pm standard error of the mean from at least three independent experiments. ISO, isoprenaline; J, juglone.

trends in the mRNA expression levels of collagen I and III, as determined using RT-qPCR analysis, were consistent with the immunofluorescence results $(\mathrm{P}<0.01$ vs. control group; $\mathrm{P}<0.01$ vs. ISO+juglone group; Fig. 3C). Taken together, these results suggested that excessive generation and deposition of collagen were associated with increased mRNA and protein levels of Pin1, whereas treatment with juglone decreased the expression of collagen I and III via the inactivation of Pin1.

Effect of Pinl on oxidative stress and antioxidant parameters in ISO-treated rats. Oxidative stress is one of the mechanisms underlying myocardial remodeling, which regulates the ECM by activating matrix metalloproteinase (MMP) and stimulating excess production of reactive oxygen species (ROS), ultimately resulting in collagen synthesis and the inflammatory response (24). In the present study, it was hypothesized that Pin1 is involved in ISO-induced cardiac fibrosis by regulating oxidative stress. To confirm this hypothesis, oxidative stress and antioxidant parameters were assessed in plasma and tissue samples using the DHE assay and biochemical analyses. Previous studies have shown that several bioactive substances are involved in the pathophysiological process of oxidative stress, among which ROS, reactive nitrogen species and lipid peroxides are crucial mediators. Conversely, MDA has been attributed to lipid peroxidation and indicates the degree of plasma membrane damage $(25,26)$. Therefore, ROS, MDA and SOD were selected for the subsequent determination of oxidative damage.

As shown in Fig. 4A, minimal ROS immunofluorescence was detected in the control group, however, its immunofluorescence was marked in the ISO group. In the ISO+juglone group, the immunofluorescence of ROS in the nuclei of proliferating cells in the myocardium was weak, compared with that in the ISO group. To further confirm the association between the expression of Pin1 and the degree of oxidative stress, the levels of MDA and SOD in the plasma and heart were evaluated. The ISO-treated rats showed significantly increased activity of SOD, an antioxidant enzyme $(\mathrm{P}<0.01)$ and increased levels of the lipid peroxidation product, MDA $(\mathrm{P}<0.01)$ in cardiac tissue, compared with the levels in the normal rats. In addition, the administration of juglone following ISO injection promoted the reduction in SOD activity $(\mathrm{P}<0.01)$ and downregulated the levels of MDA $(\mathrm{P}<0.01)$ and ROS in the heart, compared with the observations in to the ISO-treated group (Fig. 4B). Accordingly, these results confirmed that the protein level of Pin1 was also associated with oxidative stress and antioxidant substance expression during the development and progression of cardiac fibrosis, whereas the suppression of Pin1 markedly weakened the oxidative damage and occurred synchronously with heart injury. Of note, the experimental results of the biochemical analyses were consistent with the functional parameters observed on echocardiography (Fig. 2) and the 

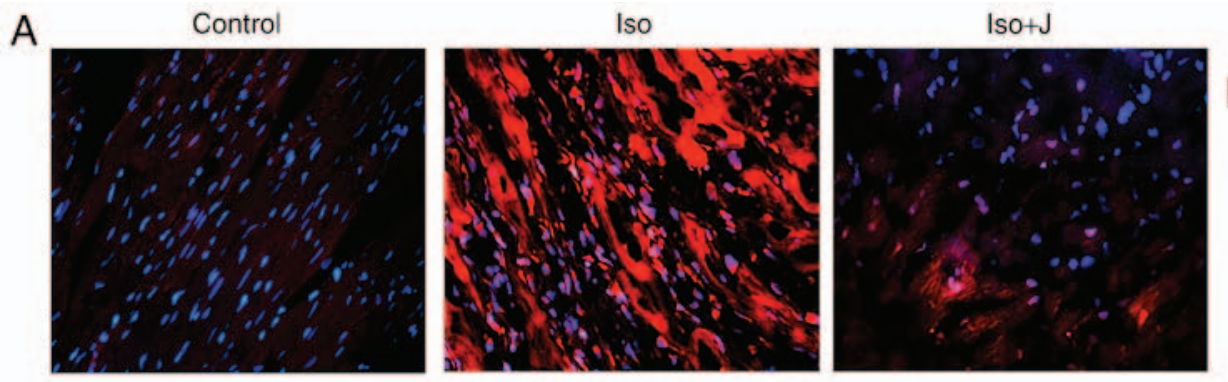

\section{B}
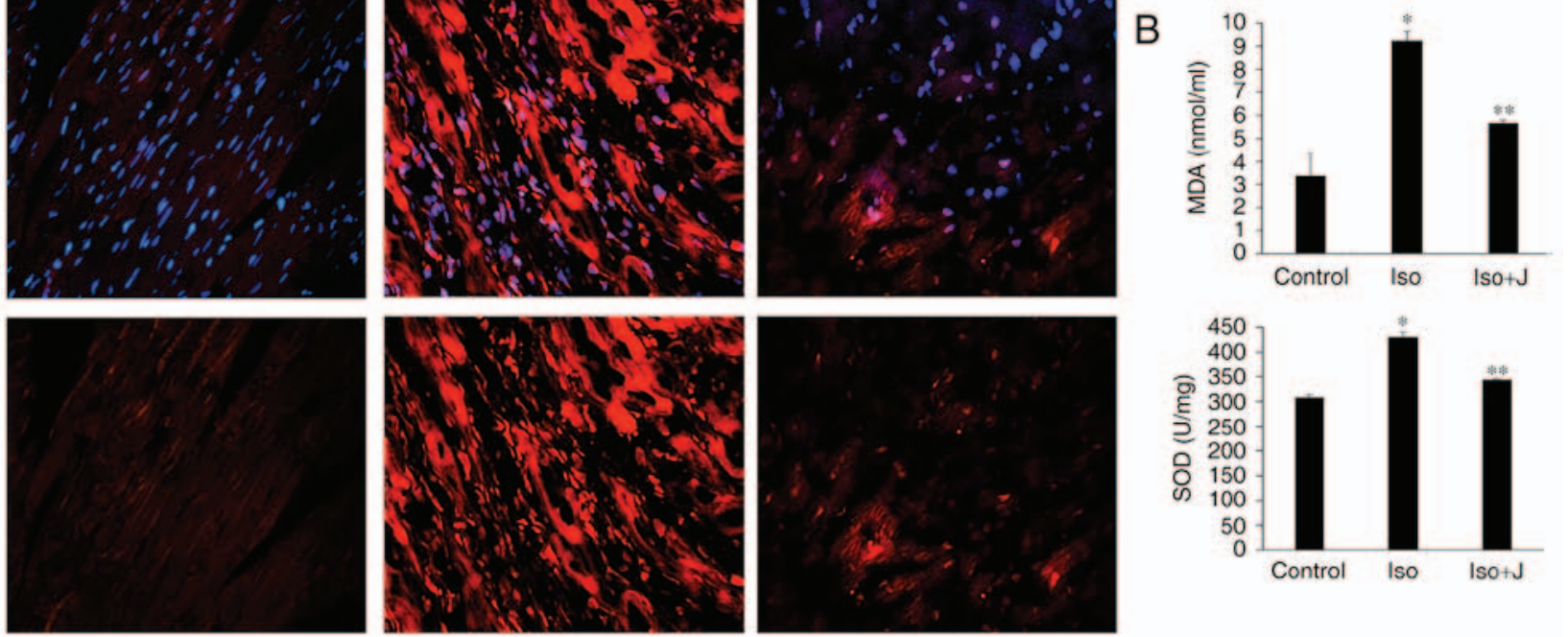

Figure 4. Oxidative stress and measurements of MDA and SOD. (A) Oxidative stress was detected using the immunofluorescent probe-DHE (magnification, $\mathrm{x} 40$ and $\mathrm{x} 200$ above and below, respectively). Reactive oxygen species were stained red and nuclei were stained blue with 4,6-diamidino-2-phenylindole (magnification, $\mathrm{x} 200$ ). (B) Expression of MDA in serum and SOD in heart tissue. ${ }^{*} \mathrm{P}<0.01 \mathrm{ISO}$ group vs. control group; ${ }^{* *} \mathrm{P}<0.01 \mathrm{ISO}$ group vs. ISO+J group. Data are presented as the mean \pm standard error of the mean from at least three independent experiments. Control, normal rat; ISO, rat intraperitoneally injected with ISO (5 mg/kg); ISO+J, ISO rat intraperitoneally injected with juglone (3 mg/kg). ISO, isoprenaline; J, juglone; MDA, malondialdehvde; SOD, superoxide dismutase.

statistical analysis of collagen deposition (Fig. 3), which also confirmed that Pin1 significantly accelerated the production of ROS and the cardiac dysfunction induced by ISO.

Pinl protein translation and activation of MEK/ERK signaling in ISO-treated rats. To examine the association among Pin1, cardiac fibrosis and dysfunction in the present study, the expression of Pin1 was evaluated in the different groups. The animal models in the ISO groups showed significantly elevated mRNA expression of Pin1 (Fig. 5) and phosphorylated protein levels of Pin1 (Fig. 6), compared with the control group. In the group treated with ISO and juglone, the protein level of pPin1 was downregulated in the heart (Fig. 6). Additionally, the increased mRNA expression of Pin1 in the ISO group $(\mathrm{P}<0.01)$ was reduced in the ISO+juglone group $(\mathrm{P}<0.01)$ (Fig. 5).

According to the results of the western blot analysis, ISO promoted the activation of $\mathrm{pERK} 1 / 2$ and protein transcription of Pin1 in response to pressure stress, and these effects were efficiently suppressed by the Pin1-specific inhibitor juglone. It was also demonstrated that ISO stimulated the expression of pMEK1/2 in vivo, which is a kinase functioning upstream from ERK1/2. Additionally, the protein expression of $\alpha$-SMA, a specialized type of protein observed in activated cardiac fibroblasts and serving as a biomarker for cardiac fibrosis, was markedly upregulated in the group of rats treated with ISO.

\section{Discussion}

Myocardial remodeling represents one of the most relevant cardiac adaptability responses to pathological hyperplasia, consisting of structural and electrical remodeling. However, microstructural analysis has shown that the structural remodeling is dependent on cardiac hypertrophy and interstitial fibrosis (27). During the initial stage of myocardial fibrosis and dysfunction, activation of the RAAS system promotes the production of catecholamines and activates the cardiac sympathetic ganglion to meet physical requirements during rest or exertion (28). However, the compensatory phase of this beneficial adaptation is irreversibly translated to the cardiac functional decompensatory period, ultimately leading to end-stage organ failure and even sudden death (29). Substantial injury and necrosis of cardiomyocytes and nonfunctional fibrous tissue increase myocardial stiffness, reduce diastolic function, and thereby affect the right and left ventricles (30). Collectively, an improved understanding of the potential mechanism underlying myocardial fibrosis is likely to enable the exploitation of novel therapeutic targets for cardiovascular diseases following various forms of damage.

Peptidyl-prolyl cis/trans isomerase is generally present in prokaryotic and eukaryotic organisms, modulating the three-dimensional conformation of phosphoproteins, and it is classified into three subfamilies: Cyclophilin, FK binding protein and parvulin. As a member of the evolutionarily conserved peptidyl-prolyl isomerase family, Pin1 was first identified and cloned from a yeast-two hybrid system in 1996. The expression of Pin 1 is mainly localized at high levels in the nucleus of the neonatal heart; following maturity, it is transferred to the cytoplasm with reduced expression. Pin1 has emerged as a novel molecule, which is involved in several complex cellular processes, including cell cycle progression, neuronal differentiation and survival, and genotoxic and cellular stress responses, and in the pathogenesis of cancer, Alzheimer's disease, Parkinson's disease and rheumatoid arthritis in humans (31). As a member of the parvulin subfamily, Pin1 has attracted interest in cardiovascular research as it catalyzes the conversion of specific pSer/Thr-Pro motifs between two completely different conformations in a subset of proteins. The functional protein acts as a signal to recruit other proteins into signaling networks or to drive substrate close to their catalytic sites, triggering numerous 
A
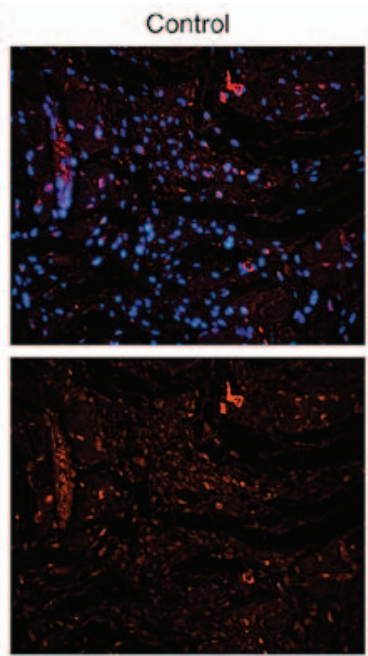
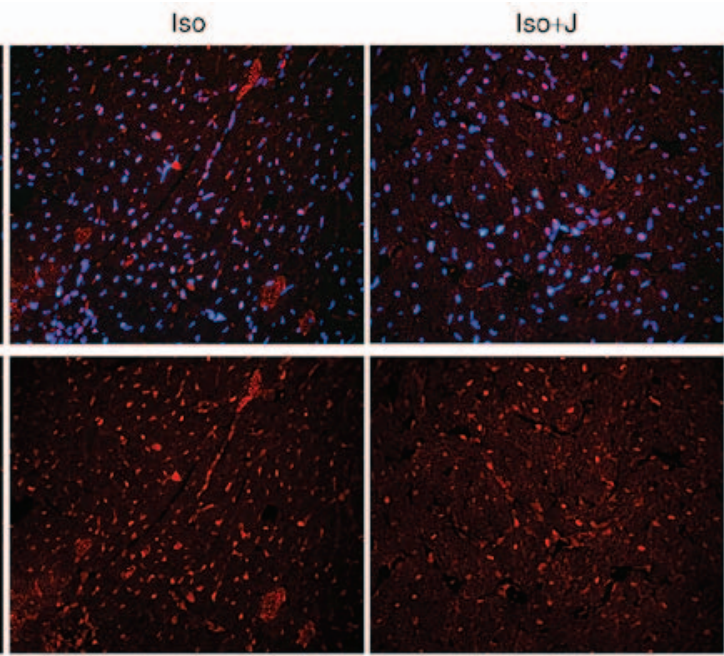
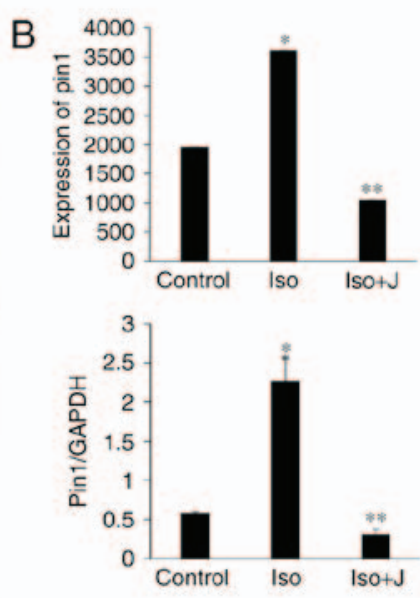

Figure 5. Expression of Pin1. (A) Representative immunostaining of Pin1. Pin1 was stained red and nuclei were stained blue with 4,6-diamidino-2-phenylindole. (B) Quantitative analysis of the expression of Pin1 via reverse transcription-quantitative polymerase chain reaction analysis and use of an associated image analysis system. GAPDH was used as the sample loading control. ${ }^{*} \mathrm{P}<0.01 \mathrm{ISO}$ group vs. control group; ${ }^{* *} \mathrm{P}<0.01 \mathrm{ISO}$ group vs. ISO+J group. Control, normal rat; ISO, rat intraperitoneally injected with ISO (5 mg/ kg); ISO+J, ISO rat intraperitoneally injected with juglone (3 mg/kg). Data are presented as the mean \pm standard error of the mean derived from at least three independent experiments. ISO, isoprenaline; J, juglone; Pin1, peptidyl-prolyl cis/trans isomerase, NIMA-interacting 1; GAPDH, glyceraldehyde phosphate dehydrogenase.

A

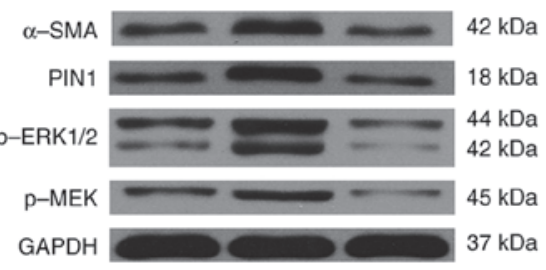

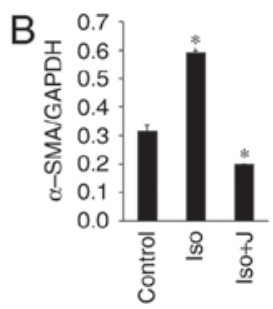

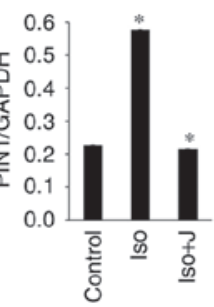

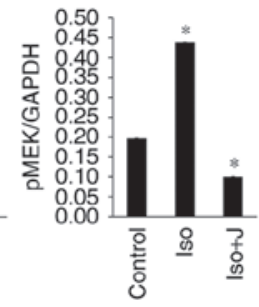

Figure 6. Expression of MEK1/2-ERK1/2 signal transduction pathway-related proteins. (A) Western blot analysis results showing the protein expression levels of $\alpha$-SMA, Pin1, pERK1/2 and pMEK. (B) Ratio of $\alpha$-SMA, Pin1, pMEK1/2 and pERK1/2 to GAPDH. *P<0.01. Control, normal rat; ISO, rat intraperitoneally injected with ISO $(5 \mathrm{mg} / \mathrm{kg})$; ISO+J, ISO rat intraperitoneally injected with juglone $(3 \mathrm{mg} / \mathrm{kg})$. Data are presented the mean \pm standard error of the mean derived from at least three independent experiments. ISO, isoprenaline; J, juglone; MEK1/2, mitogen-activated protein kinase kinase 1/2; ERK1/2, extracellular-signal regulated protein kinase 1/2; pMEK1/2, phosphorylated MEK1/2; pERK1/2, phosphorylated ERK1/2; GAPDH, glyceraldehyde phosphate dehydrogenase.

synergistic signaling cascades in the pathophysiology of cardiac tissue. As reported by Sakai et al (32), the activation of Pin1 is involved in endothelin-1 (ET-1)-induced cardiomyocyte hypertrophy by upregulating the transcriptional activity of the c-Jun N-terminal kinase signaling pathway, and by promoting hypertrophic protein synthesis, cardiomyocyte growth and cytoskeletal reorganization. However, the activity of Pin1 during cardiac fibrosis of maladaptive remodeling has not been investigated. In the present study, marked inflammatory cell infiltration, significant interstitial fibrosis and cardiac dysfunction were observed following ISO stimulation, as demonstrated by pathological examination, echocardiography and immunofluorescence, together with an increase in the protein expression of pPin1. These results suggested that the activation of Pin1 was involved in the fibrotic responses, and contributed to the structural and functional abnormalities observed in the ISO-treated rats. Following intervention with juglone, a specific inhibitor of Pin1, the formation of fibrous tissue and systolic and diastolic dysfunction were alleviated. Pin1 inhibitors, including the natural product juglone, the small molecule polyisobutylene and others, synchronously inhibit multiple signaling pathways and subsequently prevent the activation of specific kinases or phosphatases. Juglone is an active ingredient in the traditional Chinese herb Sophora japonica Ait, and possesses anti-inflammatory, antioxidant and antifibrotic activities (33). The present study also demonstrated that the protein expression of $\alpha$-SMA manifested a trend consistent with that of Pin1. Taken together, these findings suggested that the activation of Pin1 had a positive effect on the cardiac fibrosis process following cardiac injury and that juglone exerted cardioprotective effects subsequent to the inactivation of Pin1.

Accumulating evidence has shown that cardiac remodeling is triggered and affected by the simultaneous action of diverse and cross-linked signaling cascades. Pin1 is a small protein, which contains a unique $\mathrm{N}$-terminal $\mathrm{WW}$ domain and C-terminal catalytic peptidyl prolyl isomerase domain. As the only known enzyme to specifically recognize pSer/pThr-Pro motifs, Pin1 affects the transcriptional, translational and post-translational protein conformations associated with various cell signaling pathways (32). Transforming growth factor- $\beta$ (TGF- $\beta$ ), a component of a well-known profibrogenic signaling cascade, stimulates myofibroblast proliferation, migration and differentiation, and facilitates ECM synthesis, 
which has significant consequences for the pathogenesis of cardiac fibrotic alterations. Previous studies have shown that Pin1 activates Smad-dependent pathways to mediate ECM deposition in bleomycin-induced pulmonary fibrosis, based on the interaction with Smad3 and Smad6. Notably, Pin1-KO mice exhibit apparently blunted ECM production and deposition in vitro and in vivo. These observations suggest that Pin1 exacerbates collagen accumulation and aggravates airway remodeling in an animal model of lung fibrosis, which is most commonly induced by activating the Smad pathway (34). In addition, previous experimental evidence supports the hypothesis that upregulated Pin 1 contributes to the advancement of cardiac remodeling and fibrosis in diabetic mice. Consistent with the experimental results, the complicated pathogenesis of Pin1 in myocardial fibrosis and dysfunction caused by streptozotocin was found to lie principally in the phosphorylation of phosphatidylinositol 3 kinase/protein kinase B pathways, the TGF- $\beta_{1} /$ Smad pathway, MMP balance and the expression of $\alpha$-SMA (35). Therefore, multiple molecular signals and the complexity of their interactions are implicated in the fibrotic response. Although several studies have examined the association between Pin1 and cardiovascular disease, the precise mechanism underlying the involvement of Pin1 in cardiac fibrosis through non-Smad pathways has not been elucidated. Therefore, the present study concentrated on other transduction pathways in heart remodeling, with a focus on the MEK1/2-ERK1/2 signaling pathway. During activation of the Ras/Raf/MEK/ERK signaling pathway, Ras GTPase recruits and stimulates Raf kinase and then phosphorylates MEK1/2, accompanied by activated MEK1/2-induced phosphorylation of the downstream kinases ERK1/2. Once released and activated, ERK1/2 is involved in the phosphorylation of numerous cytoplasmic targets and activates multiple transcription factors in the nucleus, ultimately resulting in the reprogramming of cardiac gene expression (36). Zheng et al (37) indicated that high expression levels of constitutively active Ras activate downstream molecules in the MEK-ERK1/2 pathway, inducing disarrangement of the myocardium and interstitial fibrosis in hypertrophic cardiomyopathy. By contrast, certain studies have indicated that different mutations promote ERK1/2 pathway activation, inducing cardiac pathologies in patients with genetic disorders $(38,39)$. However, less is known about the mechanisms of Pin1 and the MEK1/2-ERK1/2 signaling pathways in cardiac fibrosis in response to ISO. The present study provides the first detailed description, to the best of our knowledge, of the specific mechanism underlying this phenomenon.

Fibrogenesis is characterized by the excessive accumulation and deposition of ECM. The proper functioning of mesenchymal components is essential for cardiovascular health, predominantly due to its antiproliferative, anti-overstretch, and antideformative properties. ECM proteins are a family of complex macromolecules, which are involved in the structural and functional dynamic equilibrium in the heart with respect to myocardial compliance, tissue differentiation and angiogenesis. Fibrillar collagens are the most abundant collagen types in the myocardium (40), exerting essential physiological control on reparative and fibrotic pathways. These fibers are expressed at relatively high levels during the pathogenesis of myocardial remodeling. Generally, there are five types of collagen subtypes in the myocardium, of which types I and
III account for $>90 \%$ of the total collagen content of the heart and are predominantly responsible for interstitial fibrosis (41). However, disturbance of the regulated balance of the secretion, synthesis and degradation of collagen metabolism leads to structural and functional impairments in remodeling hearts. A study by Shen et al (42) examined the role of Pin1 in an animal model of renal fibrosis based on a high phosphate diet (HPD) for 8-12 weeks. It was found that individual ECM genes, which are known to be involved in tissue fibrosis, including collagens I/III/V, fibronectin-1 and TGF- $\beta 1$, were significantly increased at the mRNA level in the HPD kidney, whereas low expression levels and reduced accumulation in the ECM were observed in WT and Pin1-KO mouse kidneys. These findings also showed that Pin1 was required for macrophage recruitment and chemokine expression during the inflammatory response following HPD, excluding ECM production. These results suggest that Pin1 modulates HPD-induced renal fibrosis by regulating calcium deposition, proinflammatory cytokine expression, macrophage infiltration and ECM accumulation. Similar to previous findings, the present study revealed excessive interstitial and perivascular collagen deposition in an ISO-induced model of fibrosis. Compared with the ISO group, the CVF and interstitial collagen volume fraction were significantly decreased in the ISO+juglone group, and no abnormalities were observed in the WT controls. Additionally, the expression and distribution of type III collagen was more prominent, compared with that in type I collagen in the fibrotic heart, as revealed by immunofluorescence and RT-qPCR analysis, and this effect was completely alleviated following the administration of juglone. These results are in agreement with previous studies, and demonstrated that Pin1 was required for collagen accumulation following ISO-induced cardiac dysfunction and fibrosis. Additionally, the suppression of Pin1 effectively improved the synthesis and deposition of collagen protein.

One of the most important pathological mechanisms of cardiac fibrosis is the imbalance between pro-oxidant and antioxidant defense, also known as oxidative stress, which is a crucial regulator of various heart diseases and contributes to the pathophysiology of congestive heart failure. ROS are important components of fibrotic remodeling through directly modulating the expression of ECM, disrupting the balance of substrate metabolism, and transferring the quantity and quality of the interstitial ECM. A study by Frantz et al showed that the upregulation of ROS promoted the formation of disulfide linkages, altered protein conformation and activated multiple cellular processes involved in apoptosis, necrosis and senescence (43). Given the observed data, the appropriate management of ROS is imperative for regulating oxidative stress (44). Additionally, animal experiments have shown that mitochondrial dysfunction and oxidative stress are involved in the initiation and development of left ventricular remodeling and heart failure following myocardial infarction (45). These findings suggest that oxidative damage and fibrosis interact with each other, and accelerate structural alterations and ventricular dysfunction during deteriorating cardiac remodeling. In another study, Tanaka et al attributed the ET-1 or phenylephrine-mediated overexpression of ROS in cardiomyocytes to a reactive sympathetic nervous system. Under the same stimulating conditions, ERK1/2 activity was 
also increased. Antioxidant treatment of the cardiomyocytes effectively prevented the increased production of ROS and inhibited the activation of ERK $1 / 2$. These experimental results suggest that ROS can be inhibited by inactivation of the ERK1/2 pathway (46). Xu et al showed that ERK1/2 signaling pathway activation is responsible for diabetic myocardial pathologic changes, including oxidative stress, the inflammatory response, apoptosis and remodeling (7). However, it has been demonstrated that Pin1 regulates the mitochondrial import of the $66-\mathrm{kDa}$ isoform of the growth factor adaptor Shc (p66Shc) and promotes ROS release in mitochondria, which are involved in the regulation of oxidative damage (47). However, in the phosphorylation of signaling and disease (11), the mechanism by which Pin1 coordinates the cardiac fibrosis remains to be fully elucidated. Based on the aforementioned findings, the results of the present study indicated that SOD, an important antioxidant, and MDA, acting as a biomarker for lipid peroxidation injury, were significantly increased in the remodeling heart treated with ISO, compared with those in the control and ISO+juglone groups. The rats with ISO-induced myocardial fibrosis showed enhanced ROS production based on immunofluorescence, which was consistent with the changes in the expression of different oxidative stress markers, compared with the control rats. Conversely, oxidative stress marker activity was decreased in rat plasma and tissues exposed to ISO+juglone. In addition, the phosphorylated protein levels of Pin1, MEK1/2, ERK1/2 and $\alpha$-SMA were significantly elevated in association with excessive ECM deposition and abnormal cardiac function in ISO-treated rats. Taken together, these results led to the following conclusions: Pin1 regulated diverse functional domains underlying the cardiac remodeling process, including collagen formation and degradation, molecular pathways and stress responses. The administration of juglone significantly suppressed the overexpression of ROS, effectively attenuating myocardial collagen deposition in the interstitium and improving fibrotic myocardial remodeling.

In conclusion, the results of the present study suggested that the activation of Pin1 promoted cardiac extracellular matrix deposition and oxidative stress damage by regulating the phosphorylation of the MEK1/2-ERK1/2 signal transduction pathway and the expression of $\alpha$-SMA during the fibrotic process. By contrast, restriction of the high expression levels of Pin1 by treatment with juglone, a specific inhibitor of Pin1, alleviated cardiac injury and failure in the experimental models, and prevented the subsequent cardiac fibrosis and negative effects induced by these stimuli. These findings indicated that the inhibition of Pin1 exerted cardioprotective effects and attenuated ISO-induced cardiac fibrosis, which may be an effective therapeutic option for cardiovascular diseases and heart failure.

\section{Acknowledgements}

The present study was supported by the National Nature Science Foundation of China (grant no. 81170085).

\section{References}

1. Grimaldi V, De Pascale MR, Zullo A, Soricelli A, Infante T, Mancini FP and Napoli C: Evidence of epigenetic tags in cardiac fibrosis. J Cardiol 69: 401-408, 2017.
2. Barallobre-Barreiro J, Didangelos A, Schoendube FA, Drozdov I, Yin X, Fernán dez-Caggiano M, Willeit P, Puntmann VO, Aldama-López G, Shah AM, et al: Proteomics analysis of cardiac extracellular matrix remodeling in a porcine model of ischemia/reperfusion injury. Circulation 125: 789-802, 2012.

3. Davel AP, Brum PC and Rossoni LV: Isoproterenol induces vascular oxidative stress and endothelial dysfunction via a $\mathrm{Gi}$ $\alpha$-coupled $\beta 2$-adrenoceptor signaling path way. PLoS One 9: e91877, 2014.

4. Shin E, Ko KS, Rhee BD, Han J and Kim N: Different effects of prolonged $\beta$-adren ergic stimulation on heart and cerebral artery. Integr Med Res 3: 204-210, 2014

5. Gallo S, Sala V, Gatti S and Crepaldi T: Cellular and molecular mechanisms of HG F/MET in the cardiovascular system. Clin Sci (Lond) 129: 1173-1193, 2015.

6. Wang S, Luo M, Zhang Z, Gu J, Chen J, Payne KM, Tan Y, Wang $\mathrm{Y}$, Yin $\mathrm{X}$, Zhang $\mathrm{X}$, et al: Zinc deficiency exacerbat es while zinc supplement attenuates cardiac hypertrophy in high-fat diet-induced obese mice through modulating p38 MAPK-dependent signaling. Toxicol Lett 258: 134-146, 2016.

7. Xu Z, Sun J, Tong Q, Lin Q, Qian L, Park Y and Zheng Y: The role of ERK1/2 in the development of diabetic Cardiomyopathy. Int J Mol Sci 17: E2001, 2016.

8. Zhao X, Ji J, Yu LR, Veenstra T and Wang XW: Cell cycledependent phosphorylati on of nucleophosmin and its potential regulation by peptidyl-prolyl cis/trans iso merase. $\mathrm{J}$ Mol Biochem 4: 95-103, 2015.

9. Rogals MJ, Greenwood AI, Kwon J, Lu KP and Nicholson LK: Neighboring phosphoSer-Pro motifs in the undefined domain of IRAK1 impart bivalent advantage for Pin1 binding. FEBS J 283: 4528-4548, 2016.

10. Shah M, Smolko CM, Kinicki S, Chapman ZD, Brautigan DL and Janes KA: Profiling subcellular protein phosphatase responses to coxsackievirus B3 infection of cardiomyocytes. Mol Cell Proteomics 16 (4 suppl 1): S244-S262, 2017.

11. Lu KP and Zhou XZ: The prolyl isomerase PIN1: A pivotal new twist in phosphorylation signalling and disease. Nat Rev Mol Cell Biol 8: 904-916, 2007.

12. Wulf G, Finn G, Suizu F and Lu KP: Phosphorylation-specific prolyl isomerization: Is there an underlying theme? Nat Cell Biol 7: 435-441, 2005

13. Hariharan N and Sussman MA: Pin1: A Molecular Orchestrator in the Heart.Trends Cardiovasc Med 24: 256-262, 2014.

14. Liao XH, Zhang AL, Zheng M, Li MQ, Chen CP, Xu H, Chu QS, Yang D, Lu W, Tsai TF, et al: Chemical or genetic Pin1 inhibition exerts potent anticancer activity against hepatocellular carcinoma by blocking multiple cancer-driving pathways. Sci Rep 7: 43639, 2017.

15. Shen ZJ, Esnault S, Rosenthal LA, Szakaly RJ, Sorkness RL, Westmark PR, Sandor M and Malter JS: Pin1 regulates TGF-beta1 production by activated human and murine eosinophils and contribute stoallergic lung fibrosis. J Clin Invest 118: 479-490, 2008.

16. Driver JA, Zhou XZ and Lu KP: Pin1 dysregulation helps to explain the inverse association between cancer and Alzheimer's disease. Biochim Biophys Acta 1850: 2069-2076, 2015.

17. Liou YC, Sun A, Ryo A, Zhou XZ, Yu ZX, Huang HK, Uchida T, Bronson R, Bing G, Li X, et al: Role of the prolyl isomerase Pin 1 in protecting against age-dependent neurodegeneration. Nature 424: 556-561, 2003

18. Liou YC, Zhou XZ and Lu KP: Prolyl isomerase Pin1 as a molecular switch to det ermine the fate of phosphoproteins. Trends Biochem Sci 36: 501-514, 2011.

19. Toko H, Konstandin MH, Doroudgar S, Ormachea L, Joyo E, Joyo AY, Din S, Gude NA, Collins B, Völkers M, et al: Regulation of cardiac hypertrophic signaling by prolyl isomerase Pin1. Circ Res 112: 1244-1252, 2013.

20. Toko H, Hariharan N, Konstandin MH, Ormachea L, McGregor M, Gude NA, Sundararaman B, Joyo E, Joyo AY, Collins B, et al: Differential regulation of cellular senescence and differentiation by pro lyl isomerase pin1 in cardiac progenitor cells. J Biol Chem 289: 5348-5356, 2014.

21. Carbone L: Pain management standards in the eighth edition of the Guide for the Care and Use of Laboratory Animals. J Am Assoc Lab Anim Sci 51: 322-328, 2012.

22. Cho YS, Lee SY, Kim KH and Nam YK: Differential modulations of two glyceralde hyde 3-phosphate dehydrogenase mRNAs in response to bacterial and viral cha llenges in a marine teleost Oplegnathus fasciatus (Perciformes). Fish Shellfish Immunol 25: 472-476, 2008 
23. Gauthaman KK, Saleem MT, Thanislas PT, Prabhu VV, Krishnamoorthy KK, Devaraj NS and Somasundaram JS: Cardioprotective effect of the Hibiscus rosa sinensis flo wers in an oxidative stress model of myocardial ischemic reperfusion injury in rat. BMC Complement Altern Med 6: 32, 2006.

24. Horn MA and Trafford AW: Aging and the cardiac collagen matrix: Novel mediators of fibrotic remodelling. J Mol Cell Cardiol 93: 175-185, 2016.

25. Keune WJ, Jones DR and Divecha N: PtdIns5P and Pin1 in oxidative stress signaling. Adv Biol Regul 53: 179-189, 2013

26. Paneni F, Costantino S, Castello L, Battista R, Capretti G, Chiandotto S, D'Amario D, Scavone G, Villano A, Rustighi A, et al: Targeting prolyl-isomerase Pin1 prevents mitochondrial oxidative stress and vascular dysfunction: Insights in patients with diabetes. Eur Heart J 36: 817-828, 2015.

27. Zhou S, Sun W, Zhang Z and Zheng Y: The role of Nrf2-mediated pathway in cardiac remodeling and heart failure. Oxid Med Cell Longev 2014: 260429, 2014.

28. D'Elia E, Vaduganathan M, Gori M, Gavazzi A, Butler J and Senni M: Role of biomarkers in cardiac structure phenotyping in heart failure with preserved ejection fraction: Critical appraisal and practical use. Eur J Heart Fail 17: 1231-1239, 2015.

29. Wencker D, Chandra M, Nguyen K, Miao W, Garantziotis S, Factor SM, Shirani J, Armstrong RC and Kitsis RN: A mechanistic role for cardiac myocyte apoptosis in heart failure. J Clin Invest 111: 1497-1504, 2003.

30. Hynes RO: The extracellular matrix: Not just pretty fibrils. Science 27: 326: 1216-1219, 2009.

31. Ranganathan R, Lu KP, Hunter T and Noel JP: Structural and functional analysis of the mitotic rotamase Pin1 suggests substrate recognition is phosphorylation dependent. Cell 89: 875-886, 1997

32. Sakai S, Shimojo N, Kimura T, Tajiri K, Maruyama H, Homma S, Kuga K, Mizutani T, Aonuma K and Miyauchi T: Involvement of peptidyl-prolyl isomerase Pin1 in the inhibito ry effect of fluvastatin on endothelin-1-induced cardiomyocyte hypertrophy. Life Sci 102: 98-104, 2014.

33. Van Raamsdonk JM and Hekimi S: Deletion of the mitochondrial superoxide dismu tase sod-2 extends lifespan in Caenorhabditis elegans. Plos Genet 5: e1000361, 2009.

34. Shen ZJ, Braun RK, Hu J, Xie Q, Chu H, Love RB, Stodola LA, Rosenthal LA, Szakaly RJ, Sorkness RL and Malter JS: Pin1 protein regulates Smad protein signaling and pulmonary fibrosis. J Biol Chem 287: 23294-23305, 2012.

35. Liu X, Liang E, Song X, Du Z, Zhang Y and Zhao Y: Inhibition of Pin1 alleviates myocardial fibrosis and dysfunction in STZ-induced diabetic mice. Biochem Biophys Res Commun 479: 109-115, 2016

36. Tarone G, Sbroggiò $M$ and Brancaccio M: Key role of ERK1/2 molecular scaffolds in heart pathology. Cell Mol Life Sci 70 4047-4054, 2013.
37. Zheng M, Dilly K, Dos Santos Cruz J, Li M, Gu Y, Ursitti JA Chen J, Ross J Jr, Chien KR, Lederer JW and Wang Y: Sarcoplasmic reticulum calcium defect in Ras-induced hypertrophic cardiomyopathy heart. Am J Physiol Heart Circ Physiol 286: H424-H433, 2004.

38. Purcell NH, Wilkins BJ, York A, Saba-El-Leil MK, Meloche S, Robbins J and Molkentin JD: Genetic inhibition of cardiac ERK1/2 promotes stress-induced apopto sis and heart failure but has no effect on hypertrophy in vivo. Proc Natl Acad Sci USA 104: 14074-14079, 2007.

39. Aoki Y, Niihori T, Narumi Y, Kure S and Matsubara Y: The RAS/MAPK syndromes: Novel roles of the RAS pathway in human genetic disorders. Hum Mutat 29: 992-1006, 2008.

40. Creemers EE and Pinto YM: Molecular mechanisms that control interstitial fibrosis in the pressure-overloaded heart. Cardiovasc Res 89: 265-272, 2011.

41. Reichert K, Pereira do Carmo HR, Galluce Torina A, Diógenes de Carvalho D, Carvalho Sposito A, de Souza Vilarinho KA, da Mota Silveira-Filho L, Martins de Oliveira PP and Petrucci O Atorvastatin improves ventricular remodeling after myocardial infarction by interfering with collagen metabolism. PLoS One 11: e0166845, 2016.

42. Shen ZJ, Hu J, Shiizaki K, Kuro-o M and Malter JS: Phosphateinduced renal fibrosis requires the Prolyl isomerase Pin1. PLoS One 11: e0150093, 2016.

43. Frantz S, Kelly RA and Bourcier T: Role of TLR-2 in the activation of nuclear factor kappaB by oxidative stress in cardiac myocytes. J Biol Chem 276: 5197-5203, 2001.

44. Matsushima S, Ide T, Yamato M, Matsusaka H, Hattori F, Ikeuchi M, Kubota T, Sunagawa K, Hasegawa Y, Kurihara T, et al: Over expression of mitochondrial peroxiredoxin-3 prevents left ventricular remo deling and failure after myocardial infarction in mice. Circulation 113: 1779-1786, 2006.

45. Baudino TA, Carver W, Giles W and Borg TK: Cardiac fibroblasts: Friend or foe? Am J Physiol Heart Circ Physiol 291: H1015-H1026, 2006.

46. Tanaka K, Honda M and Takabatake T: Redox regulation of MAPK pathways and cardiac hypertrophy in adult rat cardiac myocyte. J Am Coll Cardiol 37: 676-685, 2001.

47. Pinton P, Rimessi A, Marchi S, Orsini F, Migliaccio E, Giorgio M, Contursi C, Minucci S, Mantovani F, Wieckowski MR, et al: Protein kinase $\mathrm{C}$ beta and prolyl isomerase 1 regulate mitochondrial effects of the life-span determinant p66Shc. Science 315: 659-663, 2007.

This work is licensed under a Creative Commons Attribution-NonCommercial-NoDerivatives 4.0 International (CC BY-NC-ND 4.0) License. 